\title{
PURSUING ELECTRONIC HEALTH
}

\section{A UK PRIMARY HEALTH CARE PERSPECTIVE}

\author{
Mkwama Ndeti and Carlisle George
}

Middlesex University, School of Computing Science, The Burroughs, London NW4 4BT, UK

\begin{abstract}
This paper focuses on factors affecting the use of Information and communication technologies within a primary health care environment. A case study was carried out to investigate opinions and views of Primary Health Care staff on the provision of electronic health in the UK. The study found that in addition to anticipated concerns such as cost, clinician attitudes to IT, and organisational culture, staff also had concerns such as security and psychological aspects of implementing electronic health among others. The paper discusses the findings of the study and makes recommendations for best practice to ensure success in the pursuit of electronic health in the UK.
\end{abstract}

Key words: Electronic Health, Healthcare, Primary Care, NHS.

\section{INTRODUCTION}

Very few studies, have investigated the impact of the UK National Health Service (NHS) Electronic Health ${ }^{1}$ Agenda on clinicians and staff, and how its adoption will help pave the way for electronic health (e-Health). While many different factors (discussed later) could affect the pursuit of e-Health

' Electronic Health is defined as: "an emerging field in the intersection of medical informatics, public health and business, referring to health services and information delivered or enhanced through the Internet and related technologies.... also a state-of-mind, a way of thinking, an attitude, and a commitment for networked, global thinking, to improve health care locally, regionally, and worldwide by using information and communication lechnology" (Eysenbach , JMIR 2001) 
within the NHS, this paper seeks to investigate how the UK National Program for IT (NPFIT) ${ }^{2}$ agenda impacts on the different stakeholders in primary health care, and how this will affect the pursuit of e-Health within the NHS. The paper takes a case study approach with the following two objectives: (i) to establish whether the stakeholders in the provision of primary healthcare feel prepared for electronic healthcare and to look at the factors affecting the pursuit of electronic healthcare in Primary Cares; (ii) to determine the agenda that stakeholders in the provision of primary healthcare think should be given priority in the pursuit of electronic healthcare and whether this agenda ties in with the National Agenda.

\section{GOVERNMENT AND HEALTH SERVICES}

The health sector is one of the most complex and information intensive public sector organizations in the UK. The need to have this wealth of information organized and structured is imperative for the health sector to achieve better outcomes. Currently the NHS is the body charged with the provision of health services in the UK and it continues to experience many problems. Many patients have complained of long waiting times to receive medical attention, inconsistent records, bureaucratic processes, discrimination and health inequalities among others (UK National Statistics, 2005). Furthermore, the situation is heightened by the changing and increasing expectations of patients. A major challenge is for the government (and NHS) to solve these problems and meet their obligation to provide quality healthcare to citizens.

In a response to the changing expectation and needs of patients the NHS produced a modernization agenda which culminated in an Information Strategy, 1998-2005 (NHSIA, 2004) that sought to provide: lifelong electronic health records for every person in the country; round the clock online access to patient records and information about best clinical practice for all NHS clinicians; genuinely seamless care for patients through General Practitioners (GPs), hospitals and community service sharing information across the NHS Information highway; fast and convenient public access to information and care through online information services and telemedicine; and a reduction of health inequalities. In attempting to meet these ambitious objectives the NHS and government hope to provide a platform for e-Health in the UK.

2 NPFIT see: http://www.connectingforhealth.nhs.uk. It includes: care record system; electronic booking service; electronic prescriptions; and national network infrastructure.

${ }^{3}$ Primary care refers to the basic or general level of care usually given by doctors and other healthcare professionals. 


\section{THE CHALLENGE}

The success of any initiative, especially one that is technologically inclined relies on the adoption and appreciation i.e. the benefits derived from the initiative. Among some of the obvious benefits anticipated are: reduction in waiting times; a lifetime patient record that will travel with you everywhere; easy access to the patient data and information; and increased efficiency. While the perceived benefits of the NHS modernisation agenda are obvious, the success of it lies in the adoption and acceptance by those for whom it is intended. The following are the some of the issues that have shaped the perceptions and adoption of ICTs in the Health Sector.

\subsection{Medical Culture Vs Technology Culture}

There is a big problem in implementing change in a culture where power and decision making is left in the hands of a few individuals. The health sector for instance, has a lot of authority vested in one person with a wide range of responsibility for patient care. Beaver (2003) describes this as the "horizontal" nature of the medical culture. The technology culture however is very structured in its own right and Beaver (2003) goes further to describe this as the "vertical" nature of technology. Finding a middle ground for the two may prove to be an uphill task in that while the horizontal nature of the medical culture believes in sharing information, the restrictive nature of technology is guided by providing access to only those who should have access to the information.

\subsection{The Role Of Information In HealthCare}

The health sector has very complicated and rich information needs for each department. The relevance of information shared between departments is relative. The information that one department may consider important may not be important to another department. Sheaf \& Peel (1995) suggest "What information is 'true' and 'valid' becomes a contested issue prone to different interpretations more heavily influenced by organisational culture, internal politics and individuals perceptions than any objective rationale".

The amalgamation of these complex information needs could prove to be a challenge in the pursuit of e-Health. For example, the Primary Care department may consider information about a patient's ethnicity crucial whereas it may not be of relevance to the Pharmacy department. Lack of proper information in the health sector could have adverse effects on the population (Eysenbach and Jadad, 2001). 


\subsection{Cost}

Another factor that may not favour the pursuit of electronic health is that in the technology culture, technology is always changing and unfortunately these changes are industry instigated and more often than not have great cost implications. Furthermore, while the NHS assesses its priorities, other more justifiably urgent needs take precedence over IT. Benson and Neame (1994) cite an example where the cost of a new hospital such as the Chelsea and Westminster Hospital was estimated to be $£ 300$ million while investments in healthcare computing systems during the year 1992-93 came to $£ 220$ Million. From a pragmatic perspective, it appears more sensible to invest in a building a new hospital.

Cost has always been an inhibiting factor in determining the path taken by an organisation. In Great Britain much of the cost of health care is borne by the citizens through taxation. There is intensive pressure on the government to spend the taxpayers' money in ways that produce tangible benefits. A problem arises here as the benefits of ICTS may not be readily appreciated by taxpayers. However with the changing needs and expectations of the more informed and knowledgeable, citizens are slowly beginning to see the importance of ICTs in Health Care.

\subsection{Clinician Attitudes towards the Internet}

Increasingly, healthcare professionals have to deal with patients who have access to many information resources. Using the Internet, patients can easily acquire information about conditions that they perceive to have and use this information to challenge the decisions or recommendations of healthcare professionals. This has created some form of cynicism amongst healthcare professionals especially since information available on the Internet may sometimes lack integrity. Hence healthcare professionals may develop a hostile attitude towards the Internet as a resource. This may be consequential in the current hostilities towards adoption of information and communication technologies.

\subsection{Organisational Culture}

Organisational culture plays a big role in the adoption of ICTs. Bangert and Doktor (2003) argue that healthcare organisations today are designed and structured to promote efficiencies and effectiveness of a past era characterised by formal authoritarian rule and a "one best way" mentality. They argue that most implementation problems of clinical e-Health systems stem from organisational culture. Smith (2000) identifies characteristics of 
health organisations such as professional bureaucracy, individual perceptions, adhocracy and divisionalisation that could impede on the success of IT in the health sector. Benson and Neame (1994) further emphasize that the present organisational arrangements within the health care are seen as problems in their own right and thus business reengineering can help provide better goals for the health service, while keeping the needs of patients in mind.

\section{CASE STUDY METHODOLOGY}

A case study based on a Primary Care Trust (PCT) ${ }^{4}$, located in London UK, was carried out to investigate staff perceptions towards electronic healthcare in light of the study objectives (see Section 1). The PCT was assumed to be a model representation of a PCT within the specified Strategic Health Authority. The study consisted of a series of in-depth interviews (using semi structured questionnaires) conducted to gain a full understanding of the staff perceptions (attitudes and views) on e-Health.

Theoretical Sampling (Silverman 2000) was used to select some interviewees. Also, using a snowball approach one group identified and contacted other groups they felt would give important incite to the study. The groups identified for this study within the PCT stemmed from the following departments: (i) Primary Care, Information Services; (ii) Patient Advice and Liaison Services (PALS); and (iii) Complaints and Booking Services. Each of these groups was representative of the three categories identified for the study. Those involved in interacting directly with the patients (PALs, Complaints), those involved in service provision and commissioning (Primary Care) and those involved in actual information systems and Projects for primary care. (Information Services and EBooking)

Six participants were interviewed from five departments and are identified as follows: Participant (IS) -Information Services; Participants (PAL1 and PAL2) - Patient Advice and Liaison Services; Participant (Com) - Complaints; Participant (PC)- Primary Care; and Participant (EB)- EBooking. The analysis of research findings involved: transcription of the recorded interview tapes; detailed interpretation of the transcription; categorisation of the interpretation into the themes adopted for the research; and identifying whether the interview objectives were met through the information collected.

${ }^{4}$ In the UK, a Primary Care Trust provides a range of community health services and may oversee the management of several hospitals. For reasons of confidentiality the name of the Primary Care Trust where the study was carried out is withheld. 


\subsection{Interviews}

The interviews were conducted in two stages.

Stage one concentrated on the background of the specific department. Interview questions focused: the history of the department; the role of the department within the PCT; the goals of the department; what the department hoped to accomplish; and information about the key players in the department.

Stage two focused on electronic health, examining the following themes: preparedness and concern assessment; priority assessment; benefit and appeal assessment; and assessment of success factors for E-Health. This paper reports on two of the themes namely: preparedness and concern assessment, and priority assessment.

\section{RESEARCH FINDINGS}

\subsection{Preparedness and Concerns for Electronic Health}

\subsubsection{Patient Confidentiality and Security}

The biggest concern for all participants was the issue of patient confidentiality and security for electronic records. For example Participant (PALs1) remarked: "Who decides who has access to what data about the patient and how do they decide that". All participants mentioned the Data Protection Act. The majority of the participants felt that having central systems like those pledged by the NPFIT would make the NHS vulnerable to hacker attacks. Further they were not sure whether the NHS had proper secure systems in place to prevent this.

\subsubsection{Staff and Primary Care E-Health Attitudes}

The majority of participants thought it important that the staff were informed and educated on the organisational and process changes anticipated with the e-Health agenda of NPFIT.

It is of interest to note that all of the participants felt that GPs would be the hardest group to convince of the benefits of e-Health. One participant cited the example of GPs taking part in a patient profiling exercise which required them to collect all relevant information (e.g. ethnicity and religion) about the patient. The participant pointed out that the GPs had no motivation 
and lacked the time and resources to collect this information, since they did not see what benefit they would derive from the exercise.

The participants also noted that another aspect of GPs attitudes towards IT initiatives was the feeling that they (GPs) were being monitored and would therefore require them to be accountable for some of their actions. Participant (PALs2) notes: "A good example is monitoring of registration of Patients with GPs... Some feel that GPs are discriminating towards some patients so having an electronic form that would allow a patient to register himself online "anonymously "would mean that the GP would need to explain why for instance he did not accept a certain registration of a patient. It would provide a mechanism for monitoring their actions. However acceptance of these mechanisms will prove to be the greatest challenge for the NPFIT".

The majority of participants felt that many members of staff had no idea about some of the ongoing initiatives or projects and that communication was vital to the success of the NPFIT agenda. This lack of information could lead to duplication of services and impede upon the adoption of "best practises" from previous projects. Participant (IS) noted: "how do we accept a program or initiative we know nothing about?"

On staff attitudes and skills, all participants felt that the majority of the staff were adequately trained, however some cited that there still seemed to be a feeling of technophobia amongst some of their colleagues. Participant (COM) noted: "... staff needs to be informed that IT is not the enemy".

\subsubsection{History of Projects undertaken by the NHS and Government}

It was important to note that there were some participants who were cynical of the success of the NPFIT agenda. The negative attitudes towards the NPFIT agenda were attributed to the history of previous failed projects undertaken by the NHS and government. Though they were sceptical about the success of the NPFIT initiative, they seemed somehow more hopeful about the present agenda since it appeared to have sought contributions from some of the stakeholders involved. This they felt was a different approach more likely to succeed.

\subsubsection{High Expectations of NPFIT}

The majority of the participants felt that the NPFIT had set very high expectations and questioned whether they would be able to meet these objectives given the time frames presented. Participant (PALs2) noted: "...mapping of processes to patient needs and to Information technology is a Herculean task and needs to be well thought out" 
Similar observations noted that change is never painless and that there were always going to be people who would not like the changes anticipated with the NPFIT agenda, however, the participants agreed that these changes were inevitable for a more functional NHS.

\subsubsection{Other Concerns}

An interesting perspective presented by one of the participants was that NPFIT may have in some way neglected the psychological issues relating to e-Health. The participant felt that having efficient systems could possibly mean sacrificing the empathy and human emotional interaction associated with the provision of health care services. One of the words used to describe this was "impersonal". Another participant (PALs1) remarked: "How would you present or relay information relating to one who has terminal illness and make this information available to the patient"

\subsection{Priority Assessment For Electronic Health}

Upon presentation of the NPFIT agenda for e-Health all the participants felt that the Electronic Record System was most needed at the moment. To reach this decision it was important to note that the participants reflected on their own experiences not only as staff of the NHS but also as patients. They were familiar with some of the experiences that other patients encountered in light of inaccurate data or inconsistent records. The idea of having a patients' records available anywhere in the country was very appealing to all.

Regarding other priorities that participants felt were important, it was interesting to note all the participants had varied responses and had a bias towards their own departments. They cited examples of processes within their own environments which they felt should have been given priority over all other agenda items presented by the NPFIT.

The Primary Care participant, for example, felt that the department would benefit from an electronic process that would help them monitor and audit the services of the GPs in order to help them with the commissioning ${ }^{5}$ exercise. For example, if there was a baby boom they would know to commission more pre-natal or post natal services within that particular region based on the data provided through the monitoring process.

One of the participants from Information Services felt that the monitoring of inequality and diversity was important for primary care. This is currently being facilitated by a process called "patient profiling". The system is not fully functional even though it has an online presence. The participant felt it

${ }^{5}$ Commissioning refers to the process of allocation of resources and services based on need and requirement of the health population. 
should be given priority because if a patient had an appointment and required a translator or disability support, this could be arranged beforehand and the patient would be provided with these services at the time of the appointment.

The participant from Complaints felt that an online document archival system integrated with the complaints logging system would be important because currently all the complaints are stored in paper format. This would also be able to keep track of resolve times and send alerts/reminders to relevant parties required to action complaints. This kind of system would be able to study patterns and monitor trends on the nature of complaints and help the department resolve complaints quickly. The participant also believed that a system could help reduce the number of claims. The participant felt that it should be given priority because it would make the Complaints Department more efficient and pro-active compared to the reactive nature currently characteristic of the NHS.

The participants from PALS felt priority should be given to educating the public and the staff on the services available online and the different modes of access to these services. Participant (PALs2): "It's all about behavioural change and Marketing and making the patients feel that irrespective of access method the response and times of response to their queries will be the same"

The participant from E-Booking felt that offering patients choice regarding where and when they received their treatment was very important and should be given priority. It was perceived that offering patients a choice of appointment would reduce the waiting times, and Did-Not-Attends (DNAs). Participant (EB) noted: “...this means that the patient is generally happy because the patient is more in control of their health, they choose when they are coming in. For example if they have to work or have dependents they can make their choice of where to go depending on their own schedules more. It also means that patients are more likely to turn up for their appointments".

\section{DISCUSSION AND RECOMMENDATIONS}

Every patient interaction begins in Primary Care. Much of the modernisation proposed will affect the providers of Primary Health Care the most. With over 300 Million consultations in Primary Health Care annually, the importance and contribution of Primary Care in the provision of health care cannot in anyway be underestimated. The NPFIT agenda will impact on the provision of primary care services directly. The following are some recommendations for successful implementation of the NPFIT agenda. 


\subsection{Education of Staff and Knowledge Management}

A key observation made is that very few of the participants had an indepth knowledge about the NPFIT agenda. While the NPFIT agenda includes a lot of re-organisational changes, training and educating the staff on the perceived benefit of the entire agenda is crucial in its adoption and acceptance. Furthermore training can more adequately prepare users of ICT systems for the anticipated organisational and environmental changes. Training can also stimulate discussions on issues and problems that users may encounter thus increasing their knowledge and appreciation of the systems proposed. However, training alone is not enough. A knowledge management environment needs to be created such that users with deficiencies in some areas know where to obtain information, support and help from those who are well versed in the area. A knowledge base documenting 'best practice', previous problems and information on how to resolve these problems within the environment, can help increase efficiency and productivity. It would also reduce the time spent in finding a resolution to pertinent issues. Staff and users should be encouraged to disseminate information crucial to the NHS success. While saying this, the NHS should be commended on the efforts it has placed so far in the training of its staff.

\subsection{Changing Attitudes and Involvement of All}

In order to successfully implement their agenda, the NPFIT should include all stakeholders in every stage of its progression. This would provide a sense of ownership and encourage collective responsibility by all involved. In documenting the findings of the research participants, one of the key observations made was that the staff appeared to be somewhat disjointed. There were several incidences of "passing the buck": participants feeling that some of the eminent problems arising from a dysfunctional process had nothing to do with them; and participants feeling that they were not at fault for problems encountered. No one wanted to take responsibility for issues that went beyond their department. The system or NHS organisational process was blamed. It was difficult to determine whether this was indicative and reflective of the NHS organisation as a whole or was merely an issue specific to the PCT. This, however, was beyond the scope of this research. However this observation may impinge on the success of the NPFIT as a whole. For it to be successful, a collective responsibility and ownership is required. While there is a significant benefit derived in having a national infrastructure and centralization, it is important to assess the local needs and verify whether these national objectives tie in with the local agenda. More significantly, both objectives should culminate into better 
health outcomes for the patient. It would be important not to loose site of the patient at any level.

While clinician involvement is important, the NPFIT needs to look at the adoption and acceptance of the systems it is proposing. Several research studies have been done to assess the attitudes apparent in the adoption of health information systems (Morris, 2003; Lapointe et al, 2002; Gardner and Luunsgarde, 1994). While these studies have presented many findings the salient issue discussed by all is that barriers built in acceptance of new systems can only be broken through continued use of the system.

In assessing changing attitudes towards ICTs in the health sector, Chismar and Wiley-Patton (2003) in their work on applying the Technology Acceptance Model (TAM) to physicians, found that organisations needed to emphasize the usefulness of the technology to physicians while focusing on the importance and utility of technology in performing daily tasks. A study by $\mathrm{Hu}$ et al (1999) concluded that the TAM could provide a rational representation of physicians' intention to use technologies. The study also found that perceived usefulness was a key determinant in establishing the attitudes that physicians would have towards the technology while strangely perceived ease of use was not. It is recommended that the NPFIT could use the TAM to help increase the adoption and acceptance of ICTs within the NHS

\subsection{Encourage Research and Development}

While collective responsibility and ownership is extremely crucial, a mechanism needs to be put into place where some of the recommendations and ideas given about improvement in service delivery within the NHS (especially in Primary Care) can be voiced or collected. Clinician involvement is very crucial for the success of the NPFIT agenda as clinicians generally interact with the patients and have grounded experience. This means that they can provide a useful insight in the development of the NPFIT agenda on how to improve the systems. Some of the suggestions made by the participants about what issues needed to be given priority (discussed earlier) were very valuable. Furthermore, these recommendations stemmed from the experiences of the staff on the ground. If such a mechanism were put in place it would facilitate a culture of innovation and knowledge sharing. It would further provide a resource of information at both local and national level that could aide in the formulation of a strategic health policy. Building information systems based on strategic health policy is the first step towards success in e-Health. 


\subsection{Security Recommendations}

The provision of secure and safe systems by the NHS should be paramount in their agenda. Following an NHS research study on the public's view of electronic records, the biggest concern expressed by the public was the possibility of unauthorised access to medical records (NHS, 2003). The participants interviewed in this study also reiterated this point. Not much was said about how this could be achieved or what the NPFIT proposes. A solid information and security policy needs to be put in place to address these concerns. This can help avoid errors such as when the Sheffield Hospital giving 50,000 confidential gynecological records to a data processing firm that hired people off the street to transcribe unprotected data (Beaver, 2003). It must be recognised, however, that putting in place a security policy in an organisation like the NHS that has varied information access needs is indeed a complex task.

The NPFIT agenda needs to take into account the "horizontal nature" of the health sector and those involved in administering health care. One crucial aspect of security that the NHS needs to note is that in most cases unauthorised access does not necessarily stem from outside the organisation. Usually the greatest unauthorised access threats stem from within an organisation. The NPFIT needs to find a way to address this. In its security policy, the NPFIT should guarantee that whatever action an individual takes while using the systems could be traced through provision of access. Hence one can tangibly monitor access by denying or allowing a user the right to access the processes relevant to him/her duties. However this in its own right does not stop users who are granted access from misusing or divulging information about patients to others. Generally, the challenge is a grave one and the onus is upon the NPFIT to look and find ways to resolve these pertinent issues.

\subsection{Staffing Issues}

The NPFIT agenda presents new opportunities for those within the NHS to pursue careers with the Local Service Providers and National Service Providers commissioned to implement the new e-Health systems. Staff retention can be very difficult even in an organisation like the NHS that invests large sums of money in training its staff. This means that if a member of staff who is involved in a project leaves then the project may be stopped until a new person is hired. This new person will need to be trained and given an orientation period to enable him/her to start working on the project. Unfortunately organisations have little control over staff who may want to leave. However, a proper mechanism should be put in place for a smooth 
handover from one worker to the next. This issue only goes further to reiterate the importance of having a knowledge management environment.

Another approach is to ensure that the members of staff involved in projects work in teams. Consequently if one team member leaves then there will be some form of continuity in his/her absence.

\section{CONCLUSION}

In this study, participants felt that the adoption ICTs in their daily tasks could definitely improve their work performance and productivity. The NPFIT with all its teething problems is perhaps a good start. While participants' priorities showed biases towards their own departments, the interests of the entire population should be held paramount. However, it is important that the NHS look to its staff for ideas on improvements and involve all stakeholders in its planning, as this would lead to better health outcomes. Further a cohesive relationship needs to exist between all the parties involved to ensure the success of any initiative.

The study also found that with any modernisation agenda the core elements lie in realigning the organisational processes and the use of ICT to enhance the new and existent processes. ICTs within the NHS should be used as a means to the end and not an end itself.

Access to information and effective communication within the organisation will ensure participation and eventually ownership and involvement of all. The staff are an untapped resource of information for the NHS. A knowledge management environment can serve many purposes within the NHS. It can encourage innovation and lead to research and key developments within the NHS.

Should the research findings of this study be indicative of the views of the entire staff within the NHS then the NPFIT will have several difficulties in ensuring the success of its e-Health agenda if these issues and concerns are not addressed.

\section{REFERENCES}

Bangert D and Doktor R(2003) The Role of Organizational Culture in the Management of Clinical e-health Systems Proceedings of the 36th Hawaii International Conference on System Sciences (HICSS'03) 2003 IEEE The Computer Society

Beaver K (2003). Healthcare Information Systems 2nd Edition, New York, Auerbach Publications

Benson T and Neame R (1994). Healthcare Computing Essex, Longman Group Ltd. 
Chismar W G and Wiley-Patton S(2003). Does the Extended Technology Acceptance Model Apply to Physicians Proceedings of the 36th Hawaii International Conference on System Sciences (HICSS'03) IEEE-The Computer Society

Eysenbach G, Jadad AR. (2001) Consumer health informatics in the internet age. In: Evidence-based patient choice (Editors: Adrian Edwards \& Glyn Elwyn). Oxford University Press,

Eysenbach G. (2001) What is e-health? [editorial].Journal of Medical Internet Research 2001;3(2):e20 URL: http://www.jmir.org/2001/2/e20/ Accessed 12 $2^{\text {th }}$ March 2004

Gardner R M and Luunsgarde H P (1994) Evaluation of User Acceptance of a Clinical Expert System, The Journal of American Medical Informatics J Am Med Inform Assoc. Nov/Dec 1994;1:428-438.

Hu $\mathrm{P} J$ et al,(1999) Examining the Technology Acceptance Model Using Physician Acceptance of Telemedicine Technology Journal of Management Information Systems Vol. 16 No. 2, Fall 1999 pp. 91 - 112

Lapointe L, Lamothe L and Fortin J P, The Dynamics of IT Adoption in a Major Change Process in Healthcare Delivery Proceedings of the 35th Annual Hawaii International Conference on System Sciences (HICSS-35'02) 2002, IEEE

Morris L A (2003). A survey of computer use in Scottish primary care: general practitioners are no longer techno phobic but other primary care staff need better computer access Journal of Informatics in Primary Care (2003) 11: 5-11

NHS (2003). "The Public view of Electronic Health Records" http://www.dh.gov.uk/assetRoov04/05/50/46/04055046.pdf. Accessed 10th March 2004

NHSIA (2004). National Health Service Information Authority, (1998-2005) "Information for Health", http://www.nhsia.nhs.uk/def/pages/info4health/1.asp\#Notes Accessed $16^{\text {th }}$ March 2004

Sheaf, R \& Peel V.(1995) Managing Health Service Information Systems. OU Press,

Silverman D (2000). Doing Qualitative Research A Practical Handbook. London Sage Publications, pp 105

Smith J(2000). Health Management Information Systems: A handbook for Decision Makers, Philadelphia, Open University Press pp 40

UK National Statistics (2005).hut://www.performance doh.gov. uk/nhscomplaints/index.htmL. Accessed $28^{\text {th }}$ May 2005 